\title{
24-spin clusters in the mineral boleite $\mathrm{KPb}_{26} \mathrm{Ag}_{9} \mathrm{Cu}_{24} \mathrm{Cl}_{62}(\mathrm{OH})_{48}$
}

\author{
E. S. Dreier ${ }^{1,{ }^{*}}$ S. L. Holm, ${ }^{1}$ K. Lønbæk, ${ }^{1}$ U. B. Hansen, ${ }^{1}$ M. Medarde, ${ }^{2}$ I. Živković, ${ }^{3}$ P. Babkevich, ${ }^{3}$ M. Ruminy, ${ }^{4}$ N. Casati, ${ }^{5}$ \\ A. Piovano, ${ }^{6}$ S. Rols, ${ }^{6}$ G. J. Nilsen, ${ }^{6, \dagger}$ M. Boehm, ${ }^{6}$ M. Skoulatos, ${ }^{7}$ J. Schefer, ${ }^{4}$ H. M. Rønnow, ${ }^{3}$ T. Fennell, ${ }^{4, \ddagger}$ and K. Lefmann ${ }^{1}$ \\ ${ }^{1}$ Nanoscience Center, Niels Bohr Institute, University of Copenhagen, 2100 Copenhagen $\emptyset$, Denmark \\ ${ }^{2}$ Laboratory for Scientific Developments and Novel Materials, Paul Scherrer Institut, 5232 Villigen PSI, Switzerland \\ ${ }^{3}$ Laboratory for Quantum Magnetism, Institute of Physics, Ecole Polytechnique Federale de Lausanne, CH-1015 Lausanne, Switzerland \\ ${ }^{4}$ Laboratory for Neutron Scattering and Imaging, Paul Scherrer Institut, 5232 Villigen PSI, Switzerland \\ ${ }^{5}$ Swiss Light Source, Paul Scherrer Institut, 5232 Villigen PSI, Switzerland \\ ${ }^{6}$ Institut Laue-Langevin, 71 avenue des Martyrs, 38000 Grenoble, France \\ ${ }^{7}$ Heinz Maier-Leibnitz, Zentrum (MLZ) and Physics Department E21, Technische Universität München, 85748 Garching, Germany
}

(Received 29 July 2017; revised manuscript received 19 October 2017; published 17 January 2018)

\begin{abstract}
The crystal structure of the mineral boleite contains clusters of $24 S=1 / 2 \mathrm{Cu}^{2+}$ ions that have the shape of a truncated cube formed of eight trimers connected by edges. Susceptibility measurements and exact diagonalization calculations suggest that there are strong antiferromagnetic intratrimer interactions, such that effective $S=1 / 2$ degrees of freedom emerge on the trimers below $T \lesssim 100 \mathrm{~K}$. Weaker intertrimer interactions lead to the formation of a singlet ground state for these effective spins at $T \lesssim 5 \mathrm{~K}$. The clusters in boleite offer a situation similar to single molecule magnetism, accessible to both experiment and numerics, in which the interplay of quantum spins, geometric frustration, spin entanglement, and mesoscopic system size can be studied.
\end{abstract}

DOI: 10.1103/PhysRevB.97.014416

\section{INTRODUCTION}

Much progress in quantum magnetism has been made in understanding both few-body (i.e., very small clusters such as dimers and trimers) [1,2] and many-body quantum spin systems [3-5], but it is also recognized that interesting effects can occur between the two, particular to the realm of mesoscopic physics. The study of strongly interacting mesoscopic spin systems with size of order 10 atoms, usually synonymous with single molecule magnets such as the $\mathrm{Mn}_{12}$ cluster and the $\mathrm{Fe}_{8}$ wheel, has uncovered macroscopic quantum mechanical effects such as the quantum tunneling of the magnetization $[6,7]$. More recently, systems such as the $\left(\mathrm{Cr}_{7} \mathrm{Ni}\right)_{2}$ dimerized wheels have been used as model systems for studying microscopic quantum effects such as entanglement [8,9]. The combination of mesoscopic system size and strong quantum effects gives hope of applications in quantum simulation [10], information storage and processing in quantum computers $[11,12]$, and in studies of quantum coherence, entanglement, and the interaction of quantum systems with the environment (i.e., spin and oscillator baths) [13].

Quantum effects can be revealed even in mesoscopic spin systems composed of quite large classical spins, but at the many-body limit quantum effects such as fractional excitations in spin chains are sought in systems with $S=(1 / 2,1)$. A mesoscopic spin system in which microscopic quantum fluctuations are amplified by small spins (and perhaps further enhanced by geometrical frustration), appears to be an

\footnotetext{
*erik.dreier.chr@gmail.dk

${ }^{\dagger}$ Persent address: Now at ISIS Neutron and Muon Source, Science and Technology Facilities Council, Didcot OX11 0QX, United Kingdom.

†tom.fennell@psi.ch
}

interesting possibility. An example could be the $\mathrm{V}_{15}$ cluster, in which long-lived quantum coherence can be observed [14]. $\mathrm{V}_{15}$ carries an effective $S=1 / 2$ degree of freedom $\left(S_{\text {eff }}=1 / 2\right)$ on its central trimer, and the small spin value results in weak environmental coupling and long coherence times [15]. However, in terms of exchange geometry, $\mathrm{V}_{15}$ is a complicated molecule, and it appears generally to be difficult to have a highly symmetric single molecule magnet with simple interactions and geometrical frustration, such that strong (local) quantum fluctuations and geometrical frustration can be combined with mesoscopic length scales. An alternative to a molecular magnet would be to study oxide materials in which the magnetic ions form clusters. For example, in $\mathrm{La}_{4} \mathrm{Cu}_{3} \mathrm{MoO}_{12}$ a trimer of strongly interacting $\mathrm{Cu}^{2+}$ ions resides at each lattice point, forming $S_{\text {eff }}=1 / 2$ degrees of freedom. However, compared to the clusters of a molecular magnet, the trimers interact with each other relatively strongly, and form an extended (effective) spin system (as against a mesoscopic system) that undergoes a transition to a long-range ordered magnetic state at a temperature governed by the intertrimer interactions [16]. In $\mathrm{La}_{3} \mathrm{Cu}_{2} \mathrm{VO}_{9}$, planar clusters of nine sites arranged to form corner sharing triangles (similar to a fragment of the kagome lattice) form $S_{\text {eff }}=1 / 2$ degrees of freedom when all cluster sites are occupied by a $\mathrm{Cu}^{2+}$, or $S_{\text {eff }}=0$ on one third of the clusters that carry a $\mathrm{V}^{5+}$ substituent [17]. The eventual low temperature state of this system seems not to have been definitively established, but may again be expected to involve intercluster interactions on an extended lattice.

In this paper, we discuss the mineral boleite, $\mathrm{KPb}_{26} \mathrm{Ag}_{9} \mathrm{Cu}_{24}$ $\mathrm{Cl}_{62}(\mathrm{OH})_{48}$, and present evidence that it is an example of a highly frustrated mesoscopic quantum spin system that is accessible by experiment and exact diagonalization. In the remainder of the paper we describe boleite (Sec. II), our samples and brief details of their structural characterization 


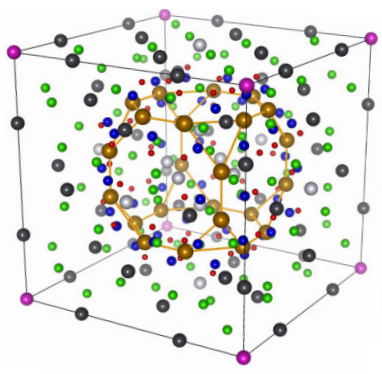

(a)

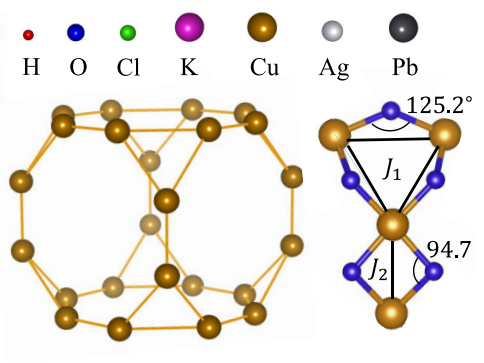

(c)
FIG. 1. The full unit cell of mineral boleite contains one formula unit of $\mathrm{KPb}_{26} \mathrm{Ag}_{9} \mathrm{Cu}_{24} \mathrm{Cl}_{62}\left(\mathrm{OH}_{48}\right)$ (a). Within this unit cell is a cluster of $\mathrm{Cu}^{2+}$ with the form of a truncated cube (b). The two closest magnetic interactions $\left(J_{1}\right.$ and $\left.J_{2}\right)$ are mediated by different $\mathrm{Cu}-\mathrm{O}$ configurations, as described in the text (c).

(Sec. III), measurements of the magnetic susceptibility and magnetization (Sec. IV), and the analysis of the susceptibility and magnetization data by numerical models (Sec. V); then we discuss the results and conclude (Sec. VI). Full details of the structural characterization (Appendix A) and various neutron scattering experiments (Appendix B) are described in appendices for interested readers.

\section{BOLEITE}

Boleite grows naturally as mm-size, deep blue crystals with the cubic space group $P m \overline{3} m$ (\#221). Boleite crystals may be zoned, with a core and exterior shell, and have epitaxial overgrowths of pseudoboleite or cumengeite [18] (both tetragonal). The core is considered to be cubic and represented by the structure that is described as follows $[18,19]$. The material has a large unit cell with the cell parameter $a=15.29 \AA$ [19]. Centered in the unit cell is a cluster of 24 $\mathrm{Cu}^{2+}$ ions, as shown in Fig. 1, well isolated from neighboring clusters by considerable quantities of diamagnetic material. The cluster is a truncated cube with eight trimers linked by cube edges. These linked trimers have a strong resemblance to the highly frustrated extended kagome lattice (also called 3-12 or star lattice) [20,21], and might be described as a small fragment of such a two-dimensional lattice, connected around periodic boundary conditions. The trimer edges have single $\mathrm{Cu}-\mathrm{O}-\mathrm{Cu}$ bridges with an angle of $125.15^{\circ}$ and $\mathrm{a} \mathrm{Cl}^{-}$anion lies on the local threefold axis bridging the whole trimer; the cube edges have two $\mathrm{Cu}-\mathrm{O}-\mathrm{Cu}$ bridges with an angle of $94.65^{\circ}$. Although the $\mathrm{Cu}-\mathrm{Cu}$ distance for the trimer edge is longer $(\approx 3.5 \AA)$ than the cube edge $(\approx 2.9 \AA)$, the $\mathrm{Cu}-\mathrm{O}$ distances are very similar in each (1.962 ̊ and $1.967 \AA$, respectively) [19] (see Fig. 1). We call the trimer edge interaction $J_{1}$ and the cube edge interaction $J_{2}$ as we expect that $J_{1}$ will be strong and antiferromagnetic while $J_{2}$ will be much weaker and close to the boundary between ferromagnetic and antiferromagnetic [22].

\section{SAMPLES AND STRUCTURAL CHARACTERIZATION}

\section{A. Samples}

We have obtained and investigated 20 natural boleite crystals, which we label A-T. Each has the typical blue color and well developed cubic form, with edges of 3-7 $\mathrm{mm}$ and masses of $0.1-0.5 \mathrm{~g}$. As will be described below, we measured the susceptibility of 20 pieces derived from 13 of the crystals and named the resulting two behaviors type I and type II. Crystals were measured in different magnetometers, and for some crystals multiple pieces of different sizes were measured. The masses and classification of all the different crystals and crystal pieces can be found in Appendix A.

\section{B. Structural characterization}

We performed various single crystal neutron and $\mathrm{x}$-ray diffraction and powder $\mathrm{x}$-ray diffraction experiments to verify that the structure of our samples is that of boleite, as described above, and by Cooper and Rouse [19]. These experiments suggest that the bulk of all of our crystals has the symmetry and structure of boleite. However, our x-ray powder diffraction experiment does suggest that other phases can occur on or near the surface of such crystals. Taken together, our structural investigations suggest that these phases are only present in small quantities. Some of our neutron diffraction measurements were made at $T=6 \mathrm{~K}$, and these reveal no structural distortion at low temperature. Full details of all these experiments are presented in Appendix A.

\section{MAGNETIC PROPERTIES}

\section{A. Magnetic susceptibility measurements}

We measured the magnetic susceptibility of the crystals using various magnetometers [Quantum Design Physical Properties Measurement System (PPMS) and Magnetic Properties Measurement System (MPMS) at PSI, PPMS at Aarhus University, MPMS and homemade ac susceptometer at EPFL]. For the measurements, large crystals were oriented with a cube edge (i.e., [l $\left.\left.\begin{array}{lll}0 & 0 & 1\end{array}\right]\right)$ approximately parallel to the magnetic field, but we assume the susceptibility to be isotropic (e.g., for comparison with unoriented fragments).

We show the susceptibility data from fifteen crystals and smaller pieces of crystals in Fig. 2(a). Two behaviors are observed: Either the susceptibility has a downturn below $T \approx 5$ K (type I) or not (type II) [Fig. 2(a) inset].

The inverse susceptibility in Fig. 2(b) shows the most striking feature of the magnetism of boleite: There are two linear regimes with a crossover at $T \approx 125 \mathrm{~K}$. The two linear regions were fitted by Curie-Weiss laws $\left[\chi^{-1}=C^{-1}(T-\right.$ $\left.\theta_{C W}\right)$ ]. Fitting in the range $170<T<250 \mathrm{~K}$ we obtain $\theta_{C W 1}=-186 \pm 6 \mathrm{~K}$ and $\mu_{1}=9.09 \pm 0.08 \mu_{B}$ f.u. $^{-1}$ (for PSI MPMS data only, as PPMS data are considerably noisier), where $\mu=\left(3 k_{B} C / N_{A}\right)^{1 / 2} / \mu_{B}$ and errors are the standard deviation amongst all the fitted values. Fitting in the range $45<T<90 \mathrm{~K}$ we obtain $\theta_{C W 2}=-9 \pm 2 \mathrm{~K}$ and $\mu_{2}=5.80 \pm$ $0.03 \mu_{B}$ f.u. $^{-1}$. It is clear that below the crossover, the moment and the magnitude of the Curie-Weiss temperature are reduced. Even at $300 \mathrm{~K}$, the magnetic moment is strongly reduced from that expected for the boleite formula unit meaning that a pure Curie-Weiss treatment does not capture the full magnetic behavior, an observation which we discuss further below. We report these values primarily as experimental quantities that can be used to compare other boleite crystals.

Some crystal pieces were excluded from further analysis. Those that were excluded exhibit a significant offset in 

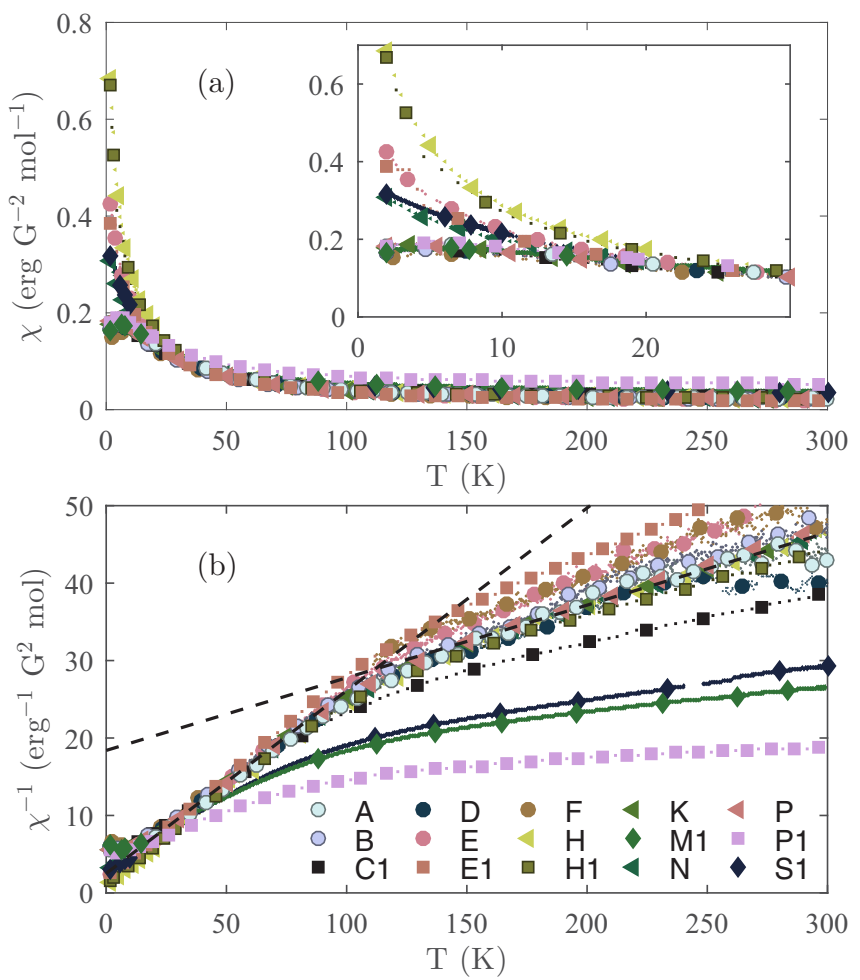

FIG. 2. The molar magnetic susceptibility (a) and inverse susceptibility (b) of fifteen boleite crystal pieces. The inset of (a) shows the two types of behavior: Type I crystals have a weak maximum, while type II do not. The inverse susceptibility in panel (b) shows two Curie-Weiss laws (dashed lines), as fitted to data from sample K. A fraction of the measured data points are shown with enlarged symbols to highlight the different crystals. The symbol shape indicates the instrument used to measure the susceptibility, as given in Appendix A.

susceptibility at high temperature $50<T<300 \mathrm{~K}$, as compared to the majority of the crystals. In fact, this selection corresponds to eliminating smaller pieces/crystals with mass below $0.03 \mathrm{~g}$. As shown in Fig. 2, when compared in absolute units the samples P1, M1, S1, C1, and E1 are clear outliers, and these are the smallest crystal pieces that are shown in the figure. It is interesting to note that the small crystal piece P1, which weighed only $0.0091 \mathrm{~g}$, behaves most differently to all the other crystals, even the large crystal $\mathrm{P}$ of which it was originally a part. However, P1, M1, S1, C1, and E1 as well as the excluded pieces still show qualitatively the same behavior as the larger pieces used for our analysis: Although the absolute magnitude of their susceptibility is different from the typical values of the large crystals, the small pieces/crystals still have the kinks in the inverse susceptibility that characterize the two boleite types, and at the same temperatures.

As suggested by reports of zoning and overgrowth in boleite crystals, and supported by our structural studies, comparing larger samples that reduce the surface area to volume ratio provides the most reproducible estimates of the magnetic properties of boleite by maximizing the signal from the core and minimizing the contribution of any near-surface impurity phases. The comparison of such samples is much more reliable, with most of the inverse susceptibilities reported in Fig. 2(b) collapsing accurately onto one another in both regimes. As a result of the above observations, for the fitting and comparison of our Hamiltonian presented in the remainder of the paper, we use only the susceptibility of the large crystals measured at PSI and the magnetization of a large piece measured at EPFL.

\section{B. Evidence of a paramagnetic contribution}

A Curie tail due to paramagnetic defects is a common feature of natural mineral samples, and it is likely that our boleite samples would show such a tail, which may obscure the intrinsic low temperature behavior. Our susceptibility measurements of type I boleite crystals suggest that the intrinsic susceptibility of boleite does drop at low temperatures but that different Curie tail contributions partially or fully obscure the peak, resulting in the various signals shown in the inset of Fig. 2(a). Furthermore, although a Curie-Weiss law can describe the inverse susceptibility of a type II crystal throughout the range $2<T<90 \mathrm{~K}$, including this temperature region in the fits of the Curie-Weiss law results in a very broad distribution of $\mu_{2}$ and $\theta_{C W 2}$ values among the crystals, despite the very reproducible data collapse seen in Fig. 2(b) for $45<T<90$ $\mathrm{K}$, suggesting that extrinsic processes begin to dominate the susceptibility of type II crystals at low temperature.

Previously, some of us have isolated such a tail by measuring the susceptibility to very low temperatures, where the tail becomes completely dominant and can be accurately fitted and subtracted to reveal the intrinsic susceptibility [23]. We made several attempts to obtain low temperature data using an $a c$ susceptometer and dilution fridge at EPFL, but due to the limited sample size that can be accommodated by this instrument and the reproducibility problems we encounter when using small crystal fragments, we were not able to obtain a good overlap between the low temperature $a c$ susceptibility and the $d c$ susceptibility measured at higher temperatures. We observed a behavior in the low temperature $a c$ susceptibility that resembles a Curie tail in both type I and II crystals, but as we are not able to match high and low temperature susceptibility data properly; the paramagnetic contribution could not be isolated in this way.

We also attempted to quantify the possible paramagnetic behavior of crystal $\mathrm{P}$ by measuring the field dependence of the $a c$ susceptibility at low temperature, $T<1.5 \mathrm{~K}$. After subtraction of a broad background, the real part of the susceptibility contains a single sharp peak, whose amplitude increases significantly as temperature is reduced (see Fig. 3). If we model the paramagnetic contribution of an ideal paramagnet with $J=S=1 / 2$ as a function of field $B$, which follows

$$
\mu(B)=N g \mu_{B} S B_{1 / 2}(x),
$$

where $N$ is the number of paramagnetic spins per unit cell and $\mathrm{B}_{1 / 2}(x)$ is the Brillouin function with $x=\frac{g \mu_{B} B}{2 k_{B} T}$, the susceptibility of a single free magnetic spin is then described by

$$
\chi_{p}=\left.\frac{d \mu(H)}{d H}\right|_{H=0}=a\left[\frac{1}{4}-\frac{1}{4} \tanh ^{2} c B\right],
$$

where $a=\frac{g^{2} \mu_{B}^{2} N}{k_{B} T}$ and $c=\frac{g \mu_{B}}{2 k_{B} T}$. Keeping $a$ and $c$ as scalable constants we find that we can fit the data reasonably well with $a=(3.8 \pm 0.1) \times 10^{4}$ and $c=2.4 \pm 0.1 \mathrm{~T}^{-1}$ for $T=0.14$ $\mathrm{K}$ and $a=(0.60 \pm 0.05) \times 10^{4}$ and $c=2.1 \pm 0.1 \mathrm{~T}^{-1}$ for $T=1.5 \mathrm{~K}$ (the unit of $a$ is arbitrary). For a true paramagnet 


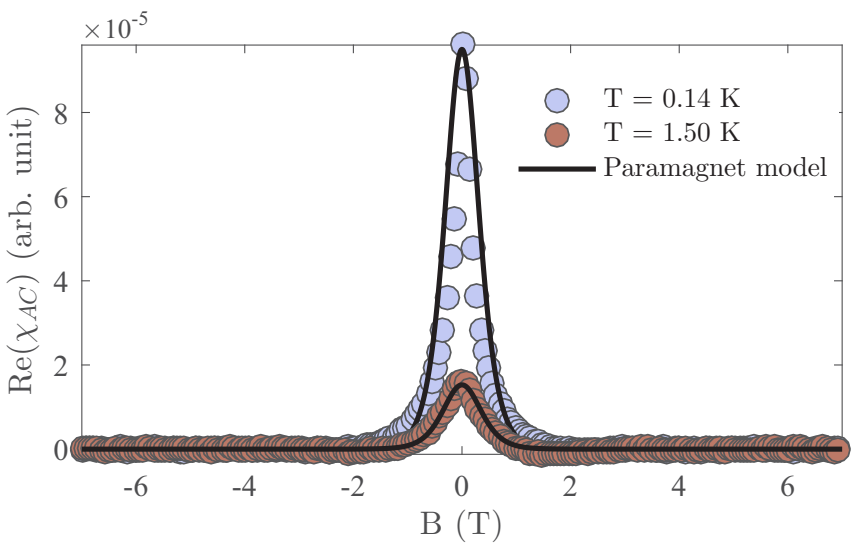

FIG. 3. The real part of magnetic $a c$ susceptibility from crystal $\mathrm{P}$ as a function of field at two different temperatures. The sharp peak can be fitted by an expression for a simple paramagnet [Eq. (2)].

we would expect both $a$ and $c$ to scale inversely with the temperature. It is therefore clear that even though we find a behavior resembling that of a paramagnet (increasing susceptibility with decreasing temperature) the relationship is not exactly that of a true paramagnet. Possible causes of this could be correlations among the paramagnetic spins at extremely low temperatures or the presence of multiple types of defects.

From these experimental investigations, we conclude that some kind of paramagnetic defects likely do exist in our boleite crystals of both type I and II. Due to the uncertainty in the low temperature data, arising from the difficulties in measuring small crystals, we have not been able to accurately isolate this contribution directly from the data. In the following, we propose a model of the intrinsic susceptibility of boleite, which also allows us to separate the paramagnetic contribution.

\section{NUMERICAL MODELING}

\section{A. The CPT model}

The form of the clusters suggests two possible simple models for the susceptibility of boleite: either trimerization due to a stronger $J_{1}$ or dimerization due to a stronger $J_{2}$. The susceptibility of isolated $S=1 / 2$ antiferromagnetic dimers and trimers can be solved analytically (e.g., Ref. [2]), and comparing these results suggest that $J_{1}$ is indeed stronger, as expected from the crystal chemistry considerations outlined in Sec. II. The inverse susceptibility of an isolated trimer can be approximated by a Curie-Weiss law at high temperature, which gives way to a Curie law with reduced moment at low temperatures. The two are separated by a distinct kink that occurs as the free spins of the trimer are bound into an $S_{\text {eff }}=1 / 2$ doublet degree of freedom and the $S_{\text {eff }}=3 / 2$ quadruplet excited states are frozen out, reducing the moment; no such feature appears in the inverse susceptibility of a dimer. Our observation of two Curie-Weiss laws and reduced moment at low temperature therefore leads us to suggest that boleite represents a cluster of coupled trimers, because the $S_{\text {eff }}=1 / 2$ degrees of freedom formed by $J_{1}$ interact via the weaker $J_{2}$ interaction the low temperature Curie law of an isolated trimer is replaced by a Curie-Weiss law.
We investigated this scenario and its ultimate ground state using several numerical models. Ignoring any other possible couplings (we discuss this approximation below), we use the Hamiltonian

$$
\mathcal{H}=J_{1} \sum_{\Delta} \mathbf{S}_{i} \cdot \mathbf{S}_{j}+J_{2} \sum_{\text {edge }} \mathbf{S}_{i} \cdot \mathbf{S}_{j} .
$$

For the 24-spin cluster, the dimensionality of the full problem $\left(>2 \times 10^{6}\right)$ precludes full matrix diagonalization. However, when $k_{\mathrm{B}} T \ll J_{1}$, we can project out the four ground states of each trimer (each with magnetization $m_{\Delta}= \pm 1 / 2$ ) and exclude the quadruplet states. We call this method the projectional model. In the following, the model is described in more details.

The ground state of a single trimer can be seen as one singlet between two of the sites and a free spin on the third [2]. In precise terms this equals

$$
|\mathrm{GS}\rangle=(\uparrow \downarrow \downarrow\rangle-|\uparrow \downarrow \uparrow\rangle) / \sqrt{2}
$$

with the third and free spin pointing up. However, this notation is not optimal, as it allows three different descriptions of ground states with free spin up, while an analysis shows that there are only two linearly independent solutions. Correcting for that by an orthonormal projection will break the symmetry between the three spins of the trimer. Instead, we describe the ground states by a cyclic combination of basis states $|\uparrow \downarrow \downarrow\rangle$ :

$$
\begin{aligned}
& \widehat{\Delta}=\left(|\downarrow \uparrow \uparrow\rangle+e^{+}|\uparrow \downarrow \uparrow\rangle+e^{-}|\uparrow \uparrow \downarrow\rangle\right) / \sqrt{3} \\
& \widehat{\Lambda}=\left(|\downarrow \uparrow \uparrow\rangle+e^{-}|\uparrow \downarrow \uparrow\rangle+e^{+}|\uparrow \uparrow \downarrow\rangle\right) / \sqrt{3},
\end{aligned}
$$

where $e^{ \pm}$is shorthand for $e^{ \pm i 2 \pi / 3}$. These two states are orthonormal, as are their two spin-down counterparts, which are notated with rotating arrows pointing down. With this notation, a two-trimer spin system will have a basis with states consisting of the outer product between ground states of the two trimers. Below are shown all basis states for a two-trimer system for the $m=1$ subspace (the two different trimers are illustrated with opposite pointing triangles):

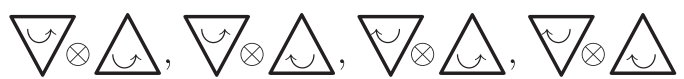

However, these states are not the eigenstates of the two-trimer system. When applying the Heisenberg Hamiltonian one finds:

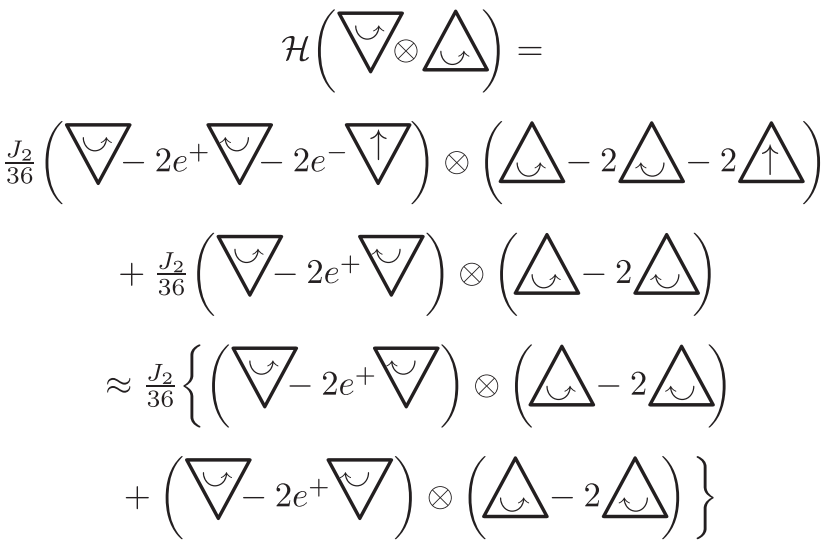



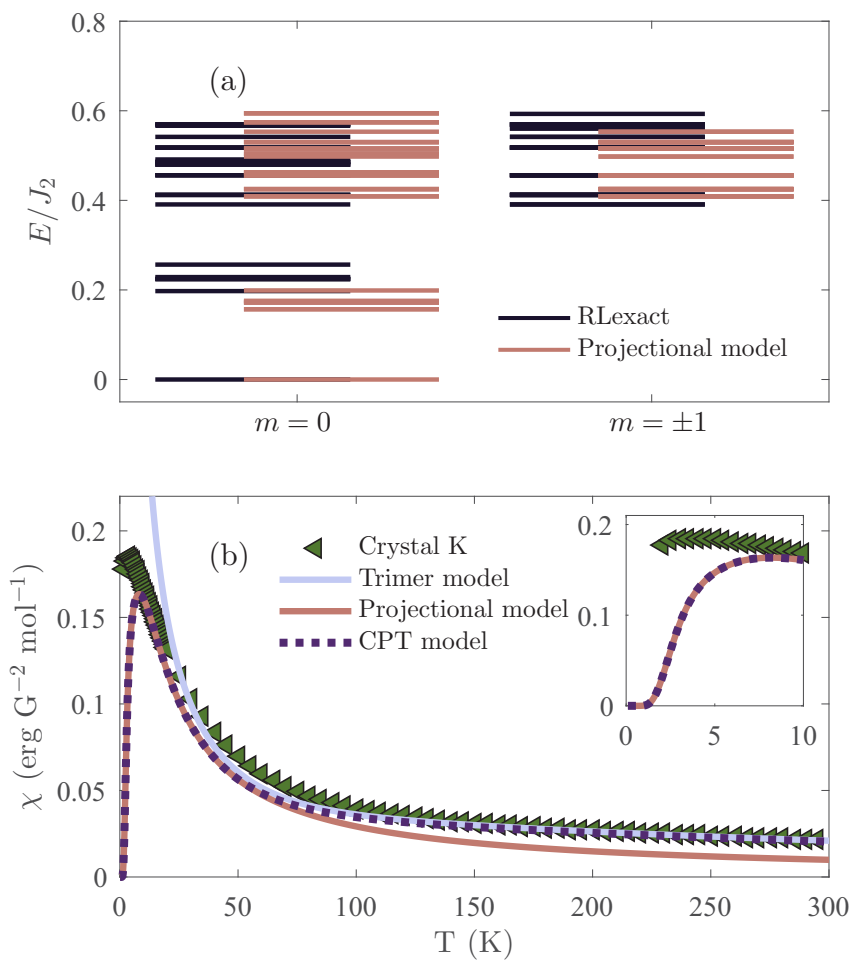

FIG. 4. Comparison of the lowest 50 excitation energies of the boleite system, as predicted by Lanczos (RLexact) and projectional model calculations (a). The susceptibility of crystal K compared to the trimer, projectional, and the combined CPT model, with $J_{1}=$ $16.8 \mathrm{meV}$ and $J_{2}=2.3 \mathrm{meV}$, with inset showing the low temperature behavior (b).

where $\uparrow$ and $\uparrow$ are the labeling for excited (quadruplet) trimer states. Excited states are removed in the projectional model, and we arrive at the final result. The model was then generalized to the full eight trimer boleite cluster, as shown in Ref. [24]. Using this model, the dimensionality of the eight-trimer problem reduces to $4^{8}=65536$ states. The projectional model states can have magnetization values of $m=-4,-3, \ldots, 4$, in contrast to the full 24-spin model that can have magnetization values of $m=-12,-11, \ldots, 12$. Since the magnetization is a good quantum number for the Heisenberg Hamiltonian, $m$ can be used to subdiagonalize the projectional model, with the largest subspace having a dimension of 17920. The resulting matrix could be diagonalized by MATLAB in $\sim 1$ hour on a powerful desktop computer, and the data was converted into susceptibility using standard statistical mechanics (i.e., $\chi=-\partial^{2} F / \partial B^{2}$ ).

The full Heisenberg model on the 24-spin cluster was further studied using the exact diagonalization program RLexact. This program employs the fourfold rotational symmetry of the system around a cubic axis, as well as the $m$ quantum number, to reduce the dimensionality of the problem to 676039 . From here, the Lanczos algorithm is employed to obtain the extreme eigenstates of the system [25,26]. In this way, the complete Hamiltonian can be probed for the important lowest eigenstates, providing a consistency check for both methods. A comparison of the lowest excitations of the projectional model and the exact method is shown in Fig. 4(a). Not all states are found by the Lanczos algorithm since it cannot distinguish degenerate states with identical symmetry properties. There is a small difference of the order $\left(J_{2} / J_{1}\right)^{2}$ between the two models, since the projectional model is a first order expansion in $J_{2} / J_{1}$. Apart from these expected differences, the agreement is good.

The projectional model effectively removes $J_{1}$ from the problem and can therefore only describe the low temperature regime of the susceptibility. Due to the expected size difference between $J_{1}$ and $J_{2}$ we suggest that a model of eight isolated trimers controlled solely by $J_{1}$ is a good approximation for the high temperature regime. To describe the full boleite system across all temperatures, we use a combined projectional and trimer model (CPT), by adding the difference between the projectional model and trimer model at $50 \mathrm{~K}$ to the trimer model. As can be seen from Fig. 4(b) (where the models contain only $J_{1}$ and $J_{2}$ as parameters and hence no scaling is done between model and data) the CPT model describes the susceptibility well for $T>10 \mathrm{~K}$ [see inset of Fig. 4(b)].

\section{B. CPT model combined with a paramagnetic contribution}

The eigenvalue spectra of both exact diagonalization and CPT calculations show a unique ground state in the singlet sector [Fig. 4(a)]. The susceptibility is expected to vanish as the system is cooled into this ground state, resulting in a peak in $\chi$ with exponentially vanishing susceptibility below the maximum. Our susceptibility measurements of type I boleite crystals suggest that the intrinsic susceptibility of boleite does drop at low temperatures, but, as discussed above, different Curie tail contributions partially or fully obscure the peak. Due to the unsuccessful attempt to isolate the Curie tail directly from experimental data, we suggest a different approach. Assuming that the CPT model is a good description of the intrinsic susceptibility of boleite, we can combine it with a Curie tail, described by Eq. 2, and fit both model and impurity parameters. It is important to stress that the fit of the Curie tail and CPT model has only $J_{1}, J_{2}$, and the defect concentration $N$ as fit parameters. From the best fit results, the Curie tail can be estimated and subtracted, revealing the intrinsic susceptibility of boleite. The procedure is shown for crystal $\mathrm{K}$ in Fig. 5, together with the resulting CPT, projectional, and trimer models of the susceptibility. A small difference is observed between model and data at low temperature $T<4 \mathrm{~K}$, indicating that our approximation of a paramagnetic contribution only from single free spins may be too simple. It should however be noted that the subtraction is problematic at low temperature due to the asymptotic behavior of our paramagnetic model.

Figure 6 shows the susceptibility of all type I and II crystals measured using the MPMS and PPMS instruments at PSI, after subtracting a Curie tail fitted as described above. The best fit parameters for the CPT model are shown in Table I. The results are very consistent for all the type I crystals, where the presence of a weak peak in the susceptibility suggests that they have smaller defect populations and less important Curie tails. For crystals of type II, which are expected (and found) to have more significant defect populations, the results are more variable, and the best fit results are less consistent between the three crystals. 


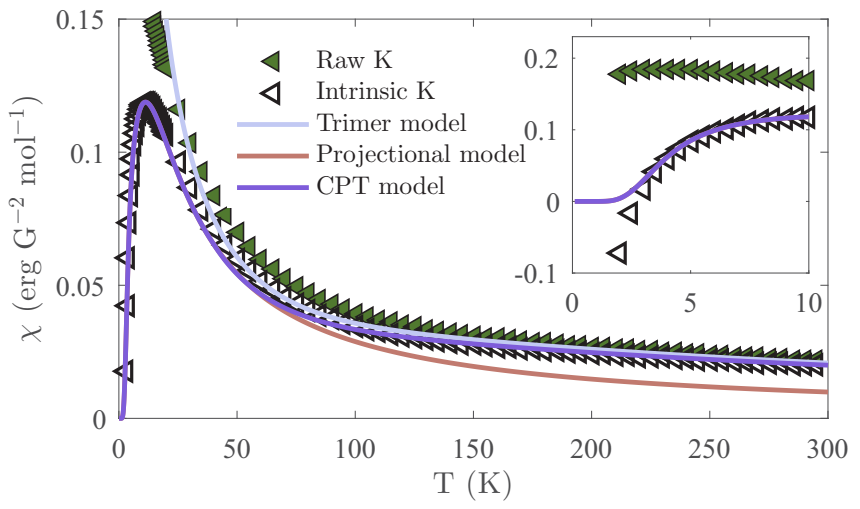

FIG. 5. The susceptibility of crystal $\mathrm{K}$ with (intrinsic K) and without (raw K) a paramagnetic contribution of 1.4 paramagnetic spins per unit cell subtracted. The resulting corrected data is compared to the projectional, trimer, and combined CPT model, $J_{1}=17.2 \mathrm{meV}$ and $J_{2}=3.2 \mathrm{meV}$, with the inset showing low temperature behavior.

The average results of fitting the models are also shown in Table I. A small difference between the results of type I and II crystals can be seen, but the values of $J_{1}$ agree within two standard deviations and for $J_{2}$ within error. The fitted defect concentration is significantly larger in type II crystals, supporting our suggestion that the difference between the two
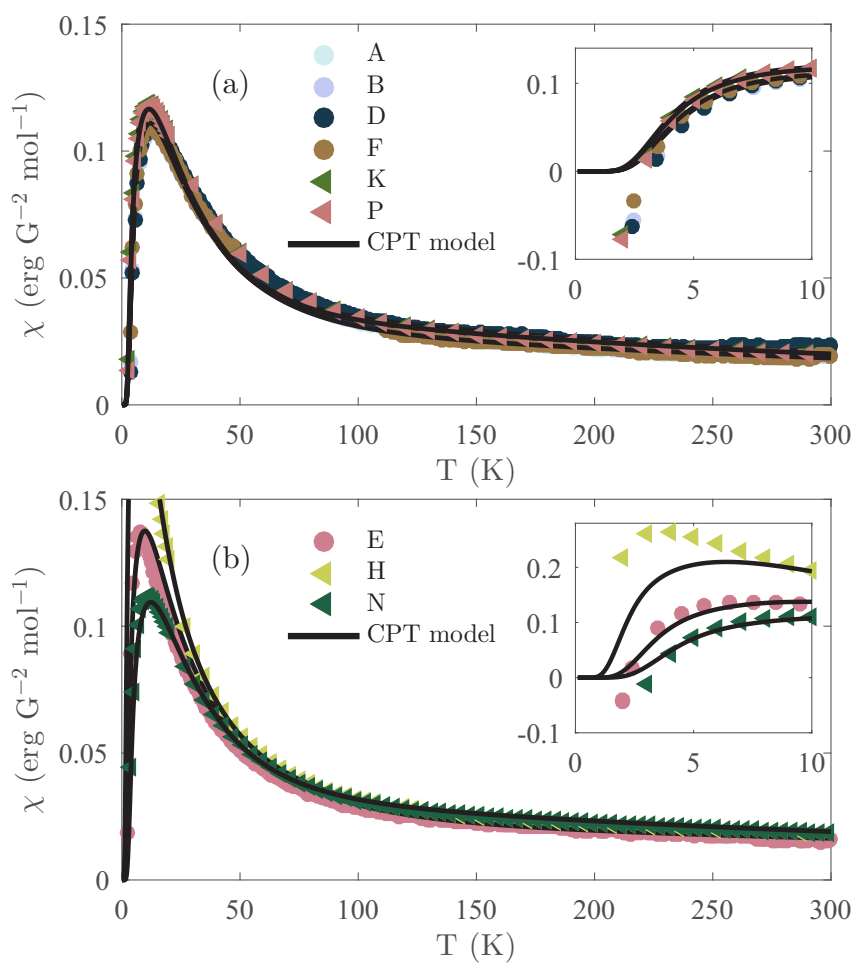

FIG. 6. The estimated intrinsic susceptibility of each type I (a) or type II (b) crystal measured using the MPMS (triangles) and PPMS (circles) at PSI, as found by fitting a combination of CPT model and Curie tail and subtracting the tail from the raw data (only every second data point is shown). The resulting data is plotted together with the relevant parametrization of the CPT model (lines). The best fit values for each crystal are given in Table I.
TABLE I. The best fit parameters from fitting the CPT model to the inverse susceptibility with a paramagnetic contribution included. The column 'Defects' shows the percentage of defects per unit cell compared to the 24 spin cluster (i.e., $N / 24 \times 100 \%$, where $N$ is the number of paramagnetic spins). The average best fit results can be seen in the last two lines.

\begin{tabular}{lcccc}
\hline \hline Type & Crystal & $J_{1}(\mathrm{meV})$ & $J_{2}(\mathrm{meV})$ & Defects $(\%)$ \\
\hline I & A & $19.0 \pm 0.8$ & $3.4 \pm 0.1$ & $6.3 \pm 0.4$ \\
I & $\mathrm{B}$ & $18.0 \pm 0.6$ & $3.5 \pm 0.1$ & $6.1 \pm 0.3$ \\
I & $\mathrm{D}$ & $15.7 \pm 0.3$ & $3.4 \pm 0.1$ & $6.2 \pm 0.2$ \\
I & $\mathrm{F}$ & $20.8 \pm 1.2$ & $3.4 \pm 0.2$ & $5.0 \pm 0.5$ \\
I & $\mathrm{K}$ & $17.2 \pm 0.4$ & $3.2 \pm 0.1$ & $5.6 \pm 0.2$ \\
I & $\mathrm{P}$ & $17.0 \pm 0.5$ & $3.2 \pm 0.1$ & $5.8 \pm 0.3$ \\
II & $\mathrm{E}$ & $27.2 \pm 1.3$ & $2.7 \pm 0.1$ & $10.3 \pm 0.4$ \\
II & $\mathrm{H}$ & $22.1 \pm 0.3$ & $1.79 \pm 0.03$ & $10.4 \pm 0.2$ \\
II & $\mathrm{N}$ & $19.2 \pm 0.1$ & $3.44 \pm 0.01$ & $9.91 \pm 0.02$ \\
I & & $17.9 \pm 1.8$ & $3.3 \pm 0.1$ & $5.8 \pm 0.5$ \\
II & & $22.8 \pm 4.0$ & $2.7 \pm 0.8$ & $10.2 \pm 0.2$ \\
\hline \hline
\end{tabular}

types is a larger amount of paramagnetic impurities in the crystals of type II.

\section{Temperature dependence of the effective magnetic moment}

The effective magnetic moment as a function of temperature can be obtained from the susceptibility data using the relation $\mu(T)=\sqrt{\left(3 k / N \mu_{B}^{2}\right) \chi T}$. The result is shown for crystal $\mathrm{K}$ and models in Fig. 7. The figure clearly illustrates the importance of the different effects at play in our models and data. At high temperatures, individual spins are free and the magnetic moment tends towards the expected value for the boleite formula unit, although given the interaction strengths we have determined, the full moment would not be recovered until $T \sim 3000 \mathrm{~K}$. The magnetic moment decreases to a plateau as $J_{1}$ drives the formation of the $S_{\text {eff }}=1 / 2$ states on the trimers, but it falls rapidly below the finite low temperature value

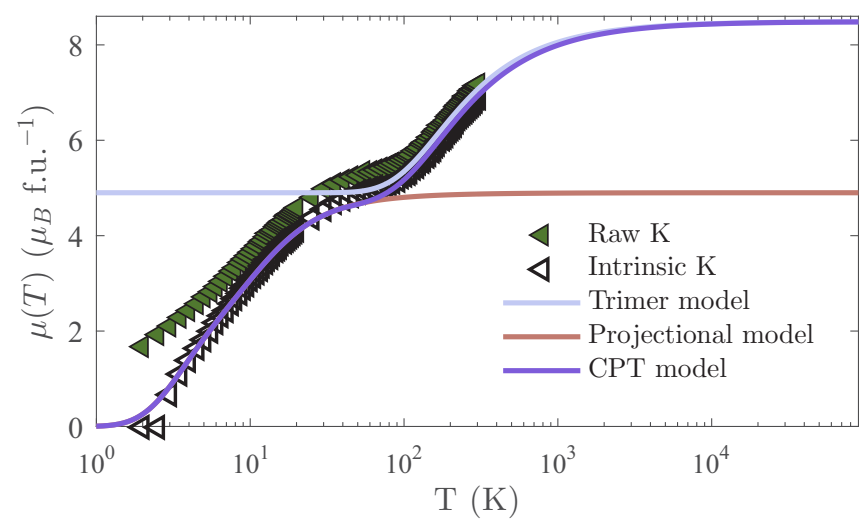

FIG. 7. The effective magnetic moment of crystal $\mathrm{K}$ calculated from the measured susceptibility [i.e., $\mu(T)=\sqrt{\left(3 k / N \mu_{B}^{2}\right) \chi T}$ ] with (intrinsic) and without (raw) contribution of 1.3 paramagnetic spins per unit cell subtracted. The corrected $\mu(T)$ is plotted together with the trimer, projectional, and CPT model with $J_{1}=17.2 \mathrm{meV}$ and $J_{2}=3.2 \mathrm{meV}$. 

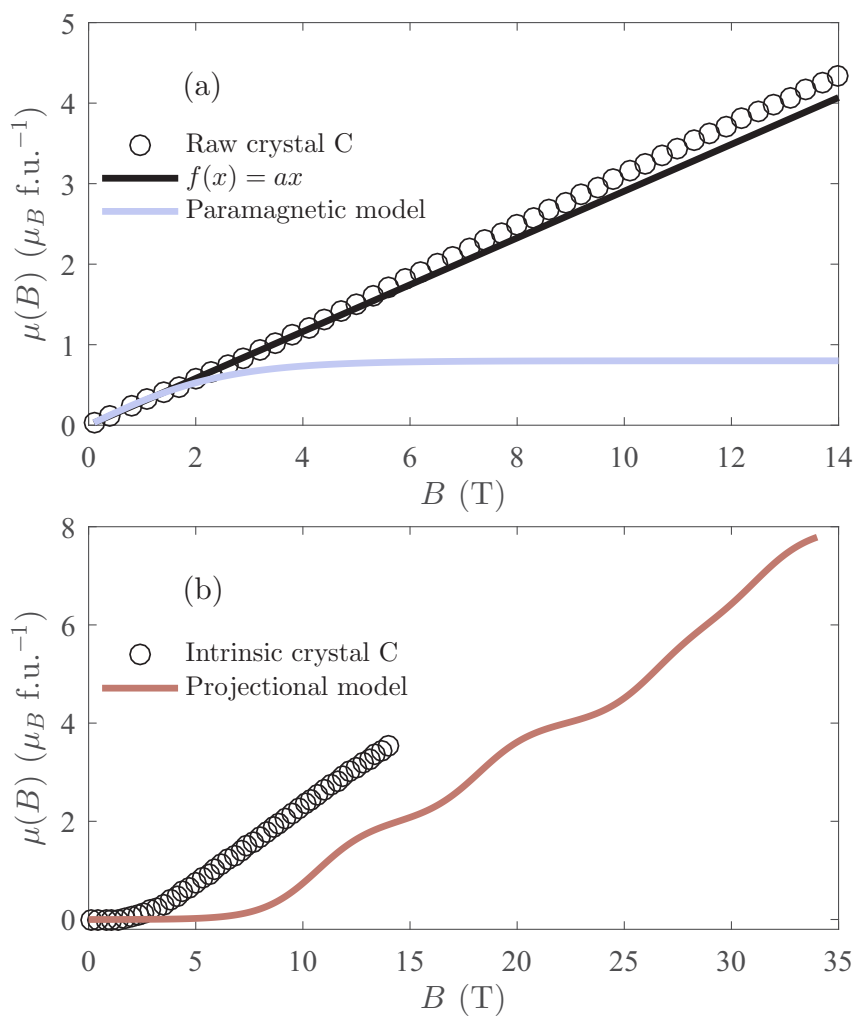

FIG. 8. Magnetization of boleite crystal $\mathrm{C} 4$ as function of applied magnetic field at $T=1.8 \mathrm{~K}$. A straight line $[f(x)=a x]$ and a paramagnetic model, described in Eq. (1), with 0.8 paramagnetic spin per unit cell are superimposed on the crystal data (a). The paramagnetic model is subtracted from the raw crystal data and the resulting intrinsic magnetization is plotted together with the projectional model for $J_{2}=3.3 \mathrm{meV}$ (b). (In these figures only every third experimental data point is shown.)

expected for isolated trimers because of the antiferromagnetic $J_{2}$ interactions, which ensure that the ground state of the full cluster is a singlet. The maximum moment of the projectional model also corresponds to the plateau, where its $S_{\text {eff }}=1 / 2$ degrees of freedom are uncorrelated.

\section{Magnetization}

The magnetization of crystal C4 was measured up to 14 $\mathrm{T}$ at $T=1.8 \mathrm{~K}$ using a PPMS with VSM at EPFL. The resulting data are shown in Fig. 8. The low field data can be fitted with a straight line. Comparing data and fit, it can be seen that the raw magnetic moment of crystal C4 increases approximately linearly from zero field and shows a small kink around $B=3 \mathrm{~T}$. The eigenvalue spectrum of Fig. 4(a) shows a gap of $\approx 1 \mathrm{meV}$ to the first states of the $m=1$ sector, which should be closed by applying a field of $\approx 7 \mathrm{~T}$. A paramagnetic contribution would therefore dominate the magnetization at low field strength and temperature. Modeling the paramagnetic contribution according to Eq. (1), we find a reasonable match to the low field magnetization of crystal $\mathrm{C} 4$ with a contribution of $N=0.8$ paramagnetic spins per unit cell. Figure $8(\mathrm{~b})$ shows the result of subtracting this paramagnetic contribution from the measured magnetization. The corrected magnetization is highly suggestive of an energy gap between ground state and the first excited $m=1$ states, but the onset of the magnetization is at $B \sim 3 \mathrm{~T}$, which is only about half of that expected.

\section{DISCUSSION}

From Fig. 6(a), it is seen that the $d c$-susceptibility data of type I boleite crystals is well represented by the combined paramagnetic and CPT model across the full temperature range $(1.7<T<300 \mathrm{~K})$. This model requires only three parameters: the two Heisenberg exchange constants and the concentration of paramagnetic spins. The fitted exchange constants are consistent, and the defect concentrations are not overwhelming. Moreover, the defect concentrations are larger in type II crystals as expected, and only for these larger paramagnetic contributions that completely obscure the peak in the raw susceptibility data does it become difficult to obtain very consistent results between different crystals. The results show that boleite can be modeled as an isolated 24-spin cluster, similar to a molecular magnet, consisting of spins strongly coupled in trimers through $J_{1}$ with weak intertrimer couplings through $J_{2}$. From our best fit result for the type I crystals, shown in Table I, we have found that on average the data is well described with $J_{1}=17.9 \pm 1.8 \mathrm{meV}$ and $J_{2}=3.3 \pm 0.1 \mathrm{meV}$. The size difference between the exchange constants means that boleite is a system in which both trimerization and the eventual formation of a unique singlet ground state from the coupled trimers can be studied.

It may be objected that we have made no successful independent verification of the model—quantities such as the effective moment are derived from the susceptibility and are necessarily well reproduced by a model that is parameterized against the susceptibility. We note that the exchange constants of the CPT model are controlled by two very robust features of the data shown in Fig. 2(b): $J_{1}$ by the temperature of the crossover between the two Curie-Weiss laws and $J_{2}$ by $\theta_{C W 2}$, both of which lie outside the temperature range where the susceptibility becomes sample dependent. The CPT model represents the simplest Hamiltonian that can reproduce these features, and if used to predict its own low temperature behavior, what remains can be fitted by a sample-dependent paramagnetic tail. The paramagnetic nature of this signal was justified independently by $a c$-susceptibility experiments. Given the nature of the samples, the experimental susceptibilities and energy scales to which they point, our model is a reasonable approximation to a realistic Hamiltonian for boleite.

Our measurement of the magnetization shown in Fig. 8(b) does constitute an independent test of the model, but there is a large discrepancy between the measured and expected onset of magnetization. We suggest that a more accurate spin Hamiltonian incorporating Dzyaloshinski-Moria exchange, for example, might reveal a mixing of $m=0,1$ states, such that the pure $m=0$ states at $\approx 0.5 \mathrm{meV}$, seen in Fig. 4(a), would contribute to the magnetization. This would reduce the onset of the expected magnetization to around $B=3$ T. Dzyaloshinski-Moria interactions are important in other materials with similar crystal chemistry and can significantly effect magnetization processes [27-29].

It is possible that long range ordering could occur due to very weak exchange interactions between the spin clusters. The size of such interactions would have to be much smaller than 
$0.3 \mathrm{meV}\left(\approx J_{2} / 10\right)$, as the $d c$-susceptibility data above $T=4$ $\mathrm{K}$ is already well described by the CPT model. Due to the paramagnetic contribution and the difficulties measuring the susceptibility at lower temperatures, such small interactions cannot be completely excluded. Similarly, structural distortions may occur at low temperature, relieving the geometric frustration of the interactions, but none is visible by a significant effect on the susceptibility down to $1.8 \mathrm{~K}$, nor in a neutron diffraction determination of the crystal structure at $6 \mathrm{~K}$, nor in any of the diffuse or inelastic neutron scattering experiments discussed in Appendix B (which reach $1.8 \mathrm{~K}$, but are not high resolution structural investigations), so we suggest that if such a transition does occur, it is both subtle and at lower temperatures than we have investigated.

Our simple numerical approximations suggest that boleite could be a model system to study entanglement between 8 $S_{\text {eff }}=1 / 2$ degrees of freedom in their collective singlet ground state. Taking $\sum_{\beta} \chi_{\beta} T / \bar{g}_{\beta}^{2}<N_{A} \mu_{B}^{2} / k_{B}(\beta=x, y, z)$ as an entanglement witness [30,31] and assuming that $\chi_{x}=\chi_{y}=\chi_{z}$, with our measurements approximating one of these, and $\bar{g} \approx 2$, we arrive at the condition $\chi T<1 / 2$ for an entangled state. $\chi T$ is closely related to $\mu(T)$ plotted in Fig. 7, so this condition corresponds to $\mu(T)<2$, implying that entanglement in the clusters of boleite becomes significant below $T \approx 10 \mathrm{~K}$.

Future studies of boleite to visualize [9] and manipulate this entanglement would be of great interest, but we note that so far all our inelastic and diffuse neutron scattering experiments have been unsuccessful due to overwhelming incoherent and phonon scattering from the hydrogen-rich natural samples (see Appendix B for details). For detailed neutron studies, deuterated samples should be synthesised if possible. In this way, it would be possible at high temperatures to observe the breaking of the trimer bonds at $E=(3 / 2) J_{1}=27 \pm 3 \mathrm{meV}$, as well as the single-triplet excitation pseudocontinuum centered at $J_{2} / 2=1 \mathrm{meV}$, which would split in a magnetic field.

\section{CONCLUSION}

We have shown that boleite is a mesoscopic spin system, whose isolated 24-spin cluster spin systems can be both measured experimentally and modeled using a simple numerically accessible Hamiltonian. Our results indicate that boleite could be an interesting system in which to study the interaction and entanglement of emergent $S_{\text {eff }}=1 / 2$ degrees of freedom at low temperatures.

\section{ACKNOWLEDGMENTS}

We thank Bruce Normand for discussions. The project has been supported by the Independent Research Fond Denmark through DANSCATT. Work at PSI was partly funded by the SNSF (Schweizerischer Nationalfonds zur Föorderung der Wissenschaftlichen Forschung) (Grants No. 200021_140862 and No. 200020_162626). Susceptibility measurements were performed at the Paul Scherrer Institute, at EPFL, and at the Department of Chemistry \& Interdisciplinary Nanoscience Center (iNANO), Aarhus University (with help from M. Christensen and M. Stingaciu). Neutron and X-ray experiments were performed at ILL, Grenoble, France; SINQ and SLS, PSI, Villigen, Switzerland.
TABLE II. Classification and mass of our boleite crystals. A number in the name signifies that it is a smaller piece of a large crystal [crystal C was originally much larger $(m=998.5 \mathrm{mg})$ but broke before measurement, hence all pieces are fragments]. The following crystals are excluded from further analysis: $*$ : Crystal $\mathrm{O}$ showed a clear type I behavior, but the susceptibility curve was only measured for $T<20 \mathrm{~K}$. $\dagger$ : The susceptibility curves of these three crystals had a significant offset from all other susceptibility curves. $\nabla: \mathrm{R}$ and $\mathrm{C} 4$ were not characterized by susceptibility measurements, but $\mathrm{R}$ was used for neutron scattering experiments and $\mathrm{C} 4$ for measurements of magnetization. The fourth column records the laboratory and instrument where the magnetic properties of the crystal were measured.

\begin{tabular}{lccc}
\hline \hline Crystal & Type & Mass $(\mathrm{mg})$ & Measured at: \\
\hline $\mathrm{A}$ & I & 355 & PSI PPMS \\
$\mathrm{B}$ & I & 424 & PSI PPMS \\
$\mathrm{D}$ & I & 605 & PSI PPMS \\
$\mathrm{F}$ & I & 287 & PSI PPMS \\
$\mathrm{K}$ & I & 263 & PSI MPMS \\
$\mathrm{P}$ & I & 236 & PSI MPMS \\
$\mathrm{C} 1$ & I & 118.8 & EPFL MPMS \\
$\mathrm{P} 1$ & I & 9.1 & EPFL MPMS \\
$\mathrm{C} 2^{\dagger}$ & I & 29.9 & AU PPMS \\
$\mathrm{C} 3^{\dagger}$ & I & 9.9 & AU PPMS \\
$\mathrm{O}^{*}$ & I & 308.9 & AU PPMS \\
$\mathrm{M} 1$ & I & 84.2 & AU PPMS \\
$\mathrm{E}$ & II & 403 & PSI PPMS \\
$\mathrm{H}$ & II & 226 & PSI MPMS \\
$\mathrm{N}$ & II & 245 & PSI MPMS \\
$\mathrm{H} 1$ & II & 197.0 & EPFL MPMS \\
$\mathrm{E} 1$ & II & 137.1 & EPFL MPMS \\
$\mathrm{S} 1$ & II & 76.4 & AU PPMS \\
$\mathrm{E} 2^{\dagger}$ & II & 21.3 & AU PPMS \\
$\mathrm{R}^{\nabla}$ & & 253 & EPFL VSM \\
$\mathrm{C} 4^{\nabla}$ & & 185.1 & \\
\hline \hline
\end{tabular}

\section{APPENDIX A: SAMPLES AND STRUCTURAL CHARACTERIZATION}

\section{Samples}

In Table II, the different crystals and crystal pieces are listed by type, together with their mass and the instrument used to measure their susceptibility.

\section{Structural characterization}

We have performed various experiments to verify that the structure of our samples is that of boleite, as described by Cooper and Rouse [19]. The minerals most commonly reported to grow on the cubic core of a boleite crystal, cumengeite and pseudoboleite both have the tetragonal space group $I 4 / \mathrm{mmm}$. Because the crystals have a well developed cubic habit, a way to distinguish cubic from tetragonal symmetry is to check for the presence of a threefold symmetry axis at the corner of the cube using x-ray Laue diffraction. All our samples showed a clear threefold symmetry axis when the incident $\mathrm{x}$-ray beam illuminated a cube corner, aligned with the local body diagonal (i.e., [111] axis) of the cube. Some representative images are shown in Fig. 9. 

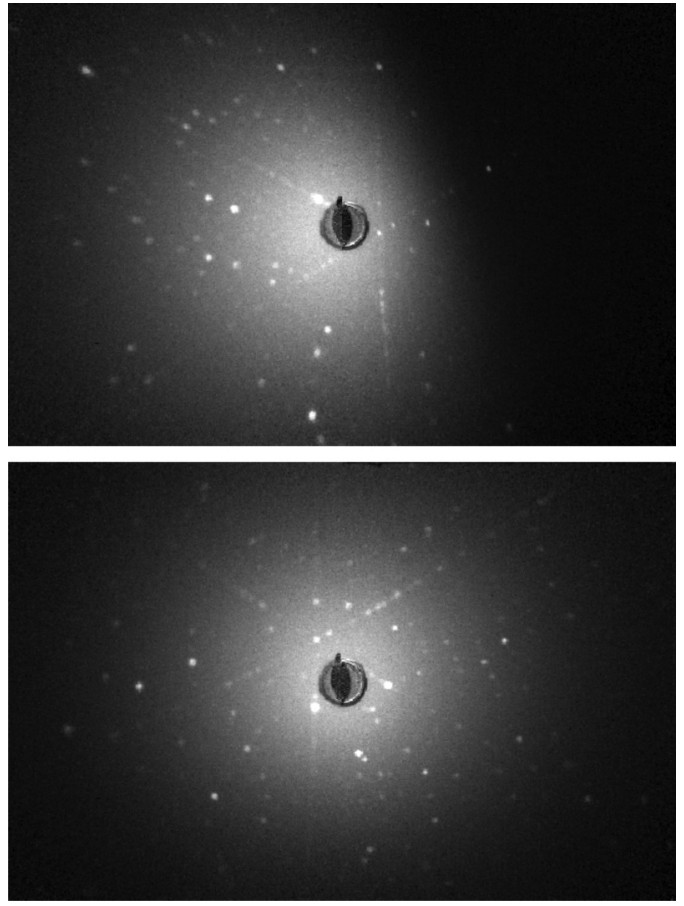

FIG. 9. Representative x-ray Laue pictures from crystals B and K.

To verify the structure, we performed synchrotron x-ray powder diffraction and single crystal neutron diffraction on a small collection of crystals. For the $\mathrm{x}$-ray powder diffraction, we used fragments scraped from the surface of crystals $\mathrm{C}$ and $\mathrm{H}$, ground together with silicon in an agate pestle and mortar, to obtain uniform powders and loaded into $0.3 \mathrm{~mm}$ glass capillaries. The silicon serves primarily to disperse the sample in the beam, while minimizing absorption, but also provides a convenient calibrant for wavelength and lattice parameters $\left(a_{\mathrm{Si}}=5.431194 \AA\right.$ at 22.5 Celcius, NIST powder diffraction standard 640c). We measured the diffraction pattern of the mixture using the high resolution powder diffractometer of the Materials Science Beamline [32] at the Swiss Light Source (SLS). The diffractometer operates in Debye-Scherrer geometry, using a Mythen microstrip detector, capillary spinner, and $2 \theta$ range extending from $2^{\circ}$ to $120^{\circ}$. The $\mathrm{x}$-ray wavelength was $\lambda \approx 0.4959 \AA$ (i.e., $E \approx 25 \mathrm{keV}$ ). All measurements were made at room temperature, which is maintained constantly at 24 Celcius at the SLS. The powder diffraction data were modeled and fitted using the Rietveld method, as implemented in the package FullProf [33]. The diffraction pattern of material scraped from sample $\mathrm{C}$ was well modeled by the structure of boleite, as shown in Fig. 10. However, material scraped from sample $\mathrm{H}$ contains a significant proportion of boleite, but also at least one other minority phase that we cannot identify (it is not the most probable candidates pseudoboleite or cumengeite).

To obtain full crystallographic data for larger samples, representative of the full volume of a crystal, we used the single crystal neutron diffractometer TriCS at SINQ to investigate three crystals: B, E, R. We used a $40^{\prime}$ collimator before the monochromator [vertical focusing PG, $\lambda=2.317(2) \AA$ ] . Before the sample we placed a PG filter $(60 \mathrm{~mm})$ and a $d=12.4 \mathrm{~mm}$ pinhole. After the sample was a $d=15 \mathrm{~mm}$
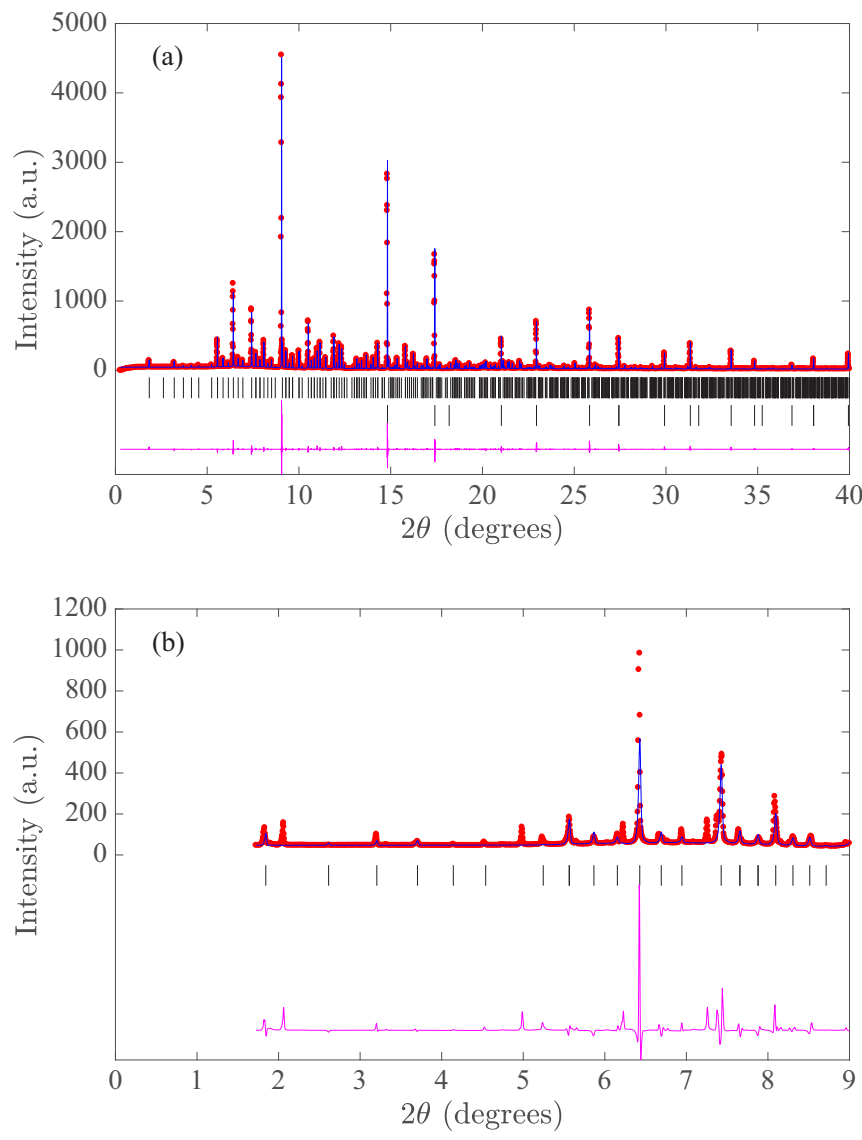

FIG. 10. Rietveld refinements of the boleite structure against synchrotron $\mathrm{x}$-ray powder diffraction data collected for scrapings of sample $\mathrm{C}$ (a) and $\mathrm{H}$ (b). Both refinements have boleite and silicon phases with peak positions indicated by ticks [no silicon peaks are within the range of panel (b)]. Although the boleite structure describes the data from sample $\mathrm{C}$ well, the unindexed peaks in the low angle part of the diffraction pattern for sample $\mathrm{H}$ shows that at least one other unidentifiable material is present.

pinhole, $80^{\prime}$ collimator, and a $45 \times 30 \mathrm{~mm}^{2}$ pinhole directly in front of the detector. The four-circle and 1D detector mode were used. The samples were mounted on aluminium pins with glue (Araldite) and attached to the cold finger of a closed cycle refrigerator. No exchange gas was used. All samples were measured at $295 \mathrm{~K}$ and additionally samples B and R were measured at $6 \mathrm{~K}$. The software package Jana2006 was used to make structural refinements.

At room temperature the crystals appear to be single crystalline boleite with cubic lattice parameter $a=15.288(2)$ $\AA$ and space group $\operatorname{Pr} \overline{3} m$. The mosaicity is low, and the $(4,0,0),(0,4,0)$, and $(0,0,4)$ reflections all have roughly the same signal strength and a Gaussian shape. The peak intensity of the $(4,0,0)$ reflection decreases by $\sim 10 \%$ as the sample is cooled. The crystal structures are well described by the boleite structure, an example of the linear correlation between the measured structure factors, and those calculated using the boleite structure is shown for sample B in Fig. 11.

In addition to the $\mathrm{x}$-ray Laue experiments (which probe only a limited volume of the sample) and the detailed crystallographic studies (that could not be carried out for all the 


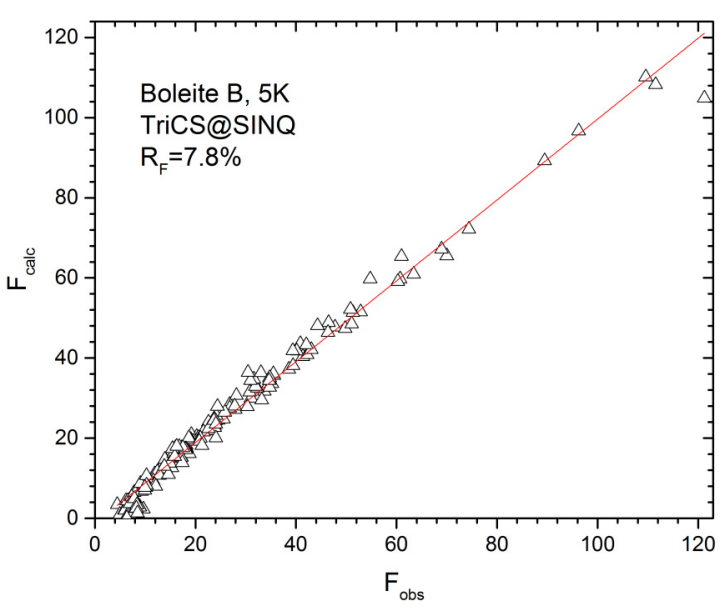

FIG. 11. Linear correlation of the measured and calculated structure factors from the refinement of the boleite structure against single crystal neutron diffraction data measured using sample (b) at $T=6 \mathrm{~K}$.

samples), to ensure that the bulk of all the larger crystals that we planned to use for neutron scattering experiments (A, B, $\mathrm{C}, \mathrm{E}, \mathrm{F}, \mathrm{H}, \mathrm{K}, \mathrm{N}$, and R) is boleite, we used the four-circle diffractometer ORION at SINQ. Cumengeite has different cell dimensions $[(a, b, c)=(15.065,15.065,24.436) \AA]$ to boleite so their diffraction patterns do not intersect. Pseudoboleite has a tetragonal cell with $(a, b, c)=(15.24,15.24,30.74) \AA$, very close in dimensions to $(1 \times 1 \times 2)$ unit cells of boleite. Pseudoboleite can be excluded by identifying the presence of all equivalents of the $(4,0,0)$ boleite reflections with roughly equal intensity (because $(4,0,0)$ and $(0,0,2)$ of pseudoboleite have quite different intensities) and the systematic absence of the strong pseudoboleite $\langle 1,0,5\rangle$ reflections (which would appear as $\langle 1,0,5 / 2\rangle$ in boleite coordinates). All crystals examined showed only the presence of boleite.

\section{APPENDIX B: NEUTRON SCATTERING EXPERIMENTS}

Apart from the diffraction measurements described above, a series of neutron experiments were conducted to investigate the magnetic structure and dynamics of boleite. These were essentially unsuccessful, but we document them here for interested readers, to avoid wasted efforts, and perhaps to inspire other/new approaches.

To examine possible high energy excitations from excited trimer states, inelastic neutron scattering experiments were made at the time-of-flight spectrometer IN4 and triple axis spectrometer IN8, with Flatcone analyser, both at the ILL. Since IN4 does not have position sensitive detectors suitable for experiments on single crystals, we continuously rotated the sample to approximate a powder average. The temperature and field dependence of the elastic structure and possible low energy excitations suggested by the projectional model were investigated at the triple axis instruments RITA-II at PSI and
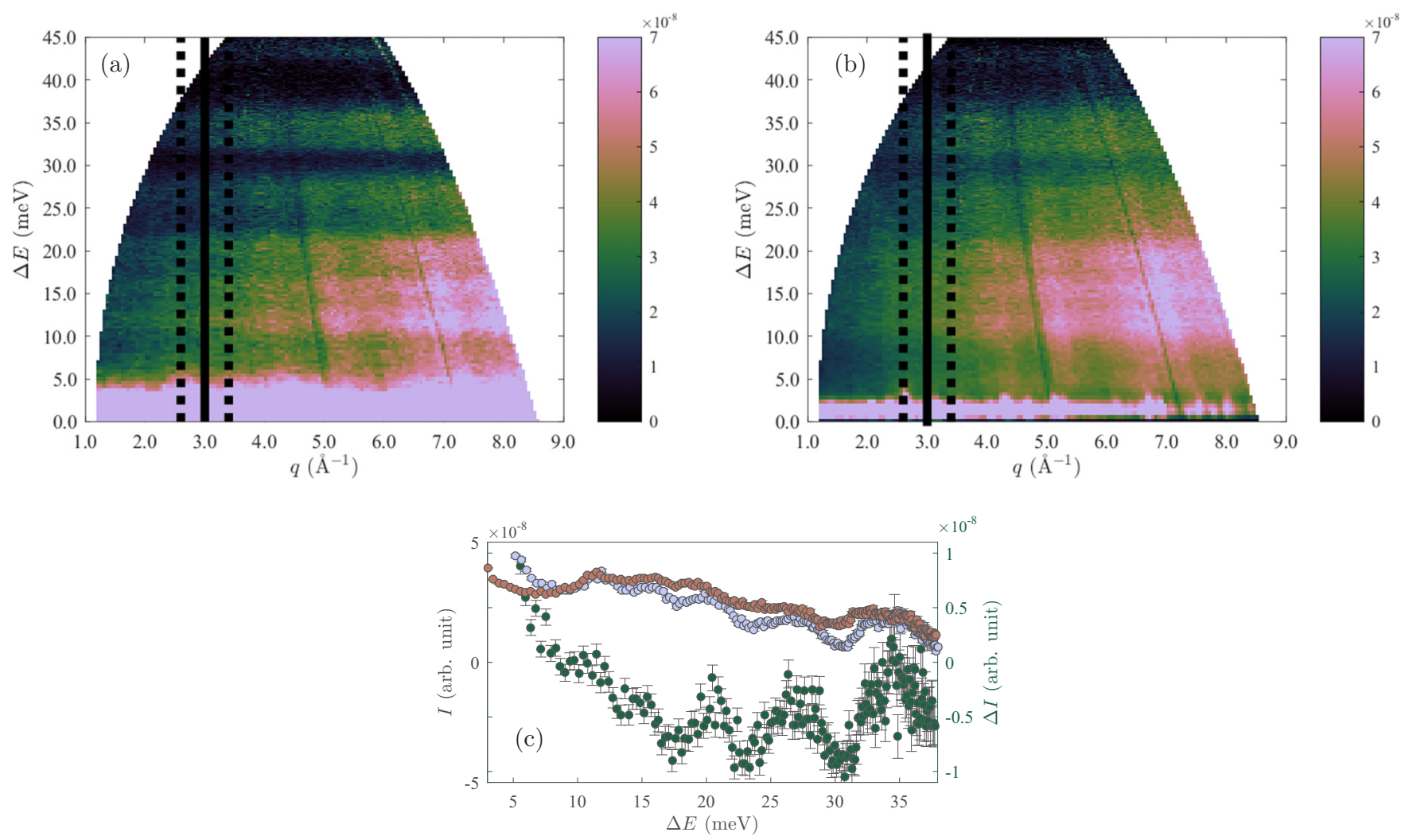

FIG. 12. Inelastic neutron scattering from boleite measured at IN4. The sample was continuously rotated through 360 degrees while measuring at $T=1.5 \mathrm{~K}$ (a) and $T=250 \mathrm{~K}$ (b). The data has been normalized with the Bose population factor. The color scales are in arbitrary units. Solid black lines mark the position of 1D cuts through the data and the dashed lines show the integration boundaries. 1D cuts at $q=3.0 \pm 0.4 \AA$ for $T=1.5 \mathrm{~K}$ (blue symbols) and $T=250 \mathrm{~K}$ (red symbols) (also with Bose factor normalization), plotted together with the difference between the two $\Delta I=I_{1.5 \mathrm{~K}}-I_{250 \mathrm{~K}}$ (green symbols, with scale on right axis) (c). 
IN3 at ILL. Neutron experiments on boleite suffer from the significant incoherent scattering cross section of hydrogen, which can create a background signal significantly larger than the magnetic scattering of the $S=1 / 2$ boleite spin system. To try to overcome this problem, the magnetic, nuclear coherent, and NSI (nuclear spin incoherent) components of the scattering signal from boleite were separated using the polarized neutron diffuse scattering spectrometer D7 at ILL.

To increase the signal several type I crystals were co-aligned along ( $\left.\begin{array}{lll}h & 0 & 0\end{array}\right)$ and $\left(\begin{array}{lll}0 & k & k\end{array}\right)$. For the first measurement of the temperature dependence of the elastic signal of boleite conducted at RITA-II, we used an aluminium sample holder which held six co-aligned crystals (A, B, D, F, K, and P) with a total crystal mass of $2165 \mathrm{mg}$. For the remaining experiments we used seven co-aligned crystals (A, B, D, F, K, O, and P) with total mass of $2474 \mathrm{mg}$, all kept in place without the use of glue.

The search for high energy excitations at the scale of $J_{1}$ [trimers should have excited states at $(3 / 2) J_{1} \sim 27 \mathrm{meV}$ ] proved to be very difficult due to the large number of optical phonon branches associated with the large unit cell of boleite. As seen in data from IN4 shown in Fig. 12, clear phonon bands are present both at low and high temperatures. Naturally, we expect the amount of magnetic scattering to decrease with increasing temperature and momentum transfer, $|q|$, while we expect the phonon scattering to increase with temperature and be proportional to $|q|^{2}$. However, due to the broadening of the phonon bands at high temperature, subtraction of the low from high temperature signal was difficult. An illustration of this problem is shown in Fig. 12(c), where it is difficult to identify any features apart from the optical phonon bands. Similar problems also prevented us from identifying a magnetic signal on IN8.

The length scale of the magnetic scattering pattern is expected to be of the same order as the reciprocal magnetic unit cell. The magnetic unit cell of boleite consists of the copper atom cluster with side length $7.15 \AA$ (compared to the structural unit cell of $a=15.288 \AA$ ). We would therefore expect the elastic scattering to be periodic along $(h, 0,0)$ with reciprocal length scale $\approx(2,0,0)$ r.l.u. However, no clear differences were seen between the high and low temperature magnetic component obtained at D7. It should also be noted that any magnetic signal is only of order $1 \%$ of the calculated NSI signal. We expect that the large NSI signal could interfere with the polarization analysis, making it difficult to isolate a small magnetic signal from a larger incoherent signal. The wave vector dependence of the NSI signal (not shown) suggests that either strong absorption (presumably due to the silver atoms) or self attenuation by the incoherent scattering further complicates these experiments. The large incoherent signal also appeared in our measurements at RITA-II and IN3, making it impossible to identify any certain evidence of magnetic signal in these experiments.
[1] A. Furrer and O. Waldmann, Rev. Mod. Phys. 85, 367 (2013).

[2] J. T. Haraldsen, T. Barnes, and J. L. Musfeldt, Phys. Rev. B 71, 064403 (2005).

[3] T. Giamarchi, C. Rüegg, and O. Tchernyshyov, Nat. Phys. 4, 198 (2008).

[4] R. Coldea, D. A. Tennant, E. M. Wheeler, E. Wawrzynska, D. Prabhakaran, M. Telling, K. Habicht, P. Smeibidl, and K. Kiefer, Science 327, 177 (2010).

[5] B. Dalla Piazza, M. Mourigal, N. B. Christensen, G. J. Nilsen, P. Tregenna-Piggott, T. G. Perring, M. Enderle, D. F. Mcmorrow, D. A. Ivanov, and H. M. Rønnow, Nat. Phys. 11, 62 (2014).

[6] L. Thomas, F. Lionti, R. Ballou, D. Gatteschi, R. Sessoli, and B. Barbara, Nature (London) 383, 145 (1996).

[7] D. Gatteschi and R. Sessoli, Angew. Chem., Int. Ed. 42, 268 (2003).

[8] A. Ardavan, O. Rival, J. J. L. Morton, S. J. Blundell, A. M. Tyryshkin, G. A. Timco, and R. E. P. Winpenny, Phys. Rev. Lett. 98, 057201 (2007).

[9] E. Garlatti, T. Guidi, S. Ansbro, P. Santini, G. Amoretti, J. Ollivier, H. Mutka, G. Timco, I. J. Vitorica-Yrezabal, G. F. S. Whitehead, R. E. P. Winpenny, and S. Carretta, Nat. Commun. 8, 14543 (2017).

[10] P. Santini, S. Carretta, F. Troiani, and G. Amoretti, Phys. Rev. Lett. 107, 230502 (2011).

[11] M. N. Leuenberger and D. Loss, Nature (London) 410, 789 (2001).

[12] G. A. Timco, S. Carretta, F. Troiani, F. Tuna, R. J. Pritchard, C. A. Muryn, E. J. L. McInnes, A. Ghirri, A. Candini, P. Santini, G. Amoretti, M. Affronte, and R. E. P. Winpenny, Nat. Nanotechnol. 4, 173 (2009).
[13] N. V. Prokof'ev and P. C. E. Stamp, Rep. Prog. Phys. 63, 669 (2000).

[14] S. Bertaina, S. Gambarelli, T. Mitra, B. Tsukerblat, A. Müller, and B. Barbara, Nature (London) 453, 203 (2008).

[15] P. C. E. Stamp and I. S. Tupitsyn, Phys. Rev. B 69, 014401 (2004).

[16] Y. Qiu, C. Broholm, S. Ishiwata, M. Azuma, M. Takano, R. Bewley, and W. J. L. Buyers, Phys. Rev. B 71, 214439 (2005).

[17] J. Robert, V. Simonet, B. Canals, R. Ballou, E. Lhotel, C. Darie, P. Bordet, B. Ouladdiaf, M. Johnson, J. Ollivier, D. Braithwaite, H. Rakoto, and S. de Brion, Phys. Rev. B 77, 054421 (2008).

[18] R. C. Rouse, J. Solid State Chem. 6, 86 (1973).

[19] M. A. Cooper and F. C. Hawthorne, Can. Mineral. 38, 801 (2000).

[20] J. H. Barry and M. Khatun, Phys. Rev. B 51, 5840 (1995).

[21] J. Richter, J. Schulenburg, A. Honecker, and D. Schmalfuss, Phys. Rev. B 70, 174454 (2004).

[22] J. B. Goodenough, Magnetism and the Chemical Bond (Wiley, New York, 1963).

[23] T. Fennell, J. O. Piatek, R. A. Stephenson, G. J. Nilsen, and H. M. Rønnow, J. Phys.: Condens. Matter 23, 164201 (2011).

[24] E. D. Christensen, MSc. thesis: Boleite, a quantum spin droplet, University of Copenhagen, 2016, http://xns.nbi.ku.dk/ student_theses/Erik_Dreier_thesis.pdf.

[25] K. Lefmann and C. Rischel, Phys. Rev. B 54, 6340 (1996).

[26] K. Lefmann and C. Rischel, Eur. Phys. J. B 21, 313 (2001).

[27] K.-Y. Choi, Y. H. Matsuda, H. Nojiri, U. Kortz, F. Hussain, A. C. Stowe, C. Ramsey, and N. S. Dalal, Phys. Rev. Lett. 96, 107202 (2006).

[28] M. Rigol and R. R. P. Singh, Phys. Rev. Lett. 98, 207204 (2007). 
[29] A. Zorko, S. Nellutla, J. van Tol, L. C. Brunel, F. Bert, F. Duc, J.-C. Trombe, M. A. de Vries, A. Harrison, and P. Mendels, Phys. Rev. Lett. 101, 026405 (2008).

[30] M. Wieśniak, V. Vedral, and Č. Brukner, New J. Phys. 7, 258 (2005).

[31] A. Candini, G. Lorusso, F. Troiani, A. Ghirri, S. Carretta, P. Santini, G. Amoretti, C. Muryn, F. Tuna, G. Timco, E. J. L. McInnes, R. E. P. Winpenny, W. Wernsdorfer, and M. Affronte, Phys. Rev. Lett. 104, 037203 (2010).
[32] P. R. Willmott, D. Meister, S. J. Leake, M. Lange, A. Bergamaschi, M. Boge, M. Calvi, C. Cancellieri, N. Casati, A. Cervellino, Q. Chen, C. David, U. Flechsig, F. Gozzo, B. Henrich, S. Jaggi-Spielmann, B. Jakob, I. Kalichava, P. Karvinen, J. Krempasky, A. Ludeke, R. Luscher, S. Maag, C. Quitmann, M. L. Reinle-Schmitt, T. Schmidt, B. Schmitt, A. Streun, I. Vartiainen, M. Vitins, X. Wang, and R. Wullschleger, J. Synchrotron Radiat. 20, 667 (2013).

[33] J. Rodriguez-Carvajal, Physica B 192, 55 (1993). 